\title{
Formation of ultra-compact blue dwarf galaxies and their evolution into nucleated dwarfs
}

\author{
Kenji Bekki \\ ICRAR, M468, The University of Western Australia 35 Stirling Highway, Crawley \\ Western Australia, 6009, Australia
}

\begin{abstract}
We propose that there is an evolutionary link between ultra-compact blue dwarf galaxies (UCBDs) with active star formation and nucleated dwarfs based on the results of numerical simulations of dwarf-dwarf merging. We consider the observational fact that low-mass dwarfs can be very gas-rich, and thereby investigate the dynamical and chemical evolution of very gas-rich, dissipative dwarf-dwarf mergers. We find that the remnants of dwarf-dwarf mergers can be dominated by new stellar populations formed from the triggered starbursts and consequently can have blue colors and higher metallicities $\left(Z \sim[0.2-1] Z_{\odot}\right)$. We also find that the remnants of these mergers can have rather high mass-densities $\left(10^{4} M_{\odot} \mathrm{pc}^{-3}\right)$ within the central $10 \mathrm{pc}$ and small half-light radii $(40-100 \mathrm{pc})$. The radial stellar structures of some merger remnants are similar to those of nucleated dwarfs. Star formation can continue in nuclear gas disks $(R<100$ pc) surrounding stellar galactic nuclei (SGNs) so that the SGNs can finally have multiple stellar populations with different ages and metallicities. These very compact blue remnants can be identified as UCBDs soon after merging and as nucleated dwarfs after fading of young stars. We discuss these results in the context of the origins of metal-rich ultra-compact dwarfs (UCDs) and SGNs.
\end{abstract}

Subject headings: galaxies: dwarf — galaxies: stellar nuclei — galaxies: star clusters - galaxies: structure — globular clusters: general

\section{Introduction}

Since the discovery of ultra-compact dwarf galaxies ("UCDs") in the Fornax clusters (Drinkwater et al. 2003), the origin of UCDs has been extensively discussed both observationally and theoretically (e.g., Bekki et al. 2003; Jones et al. 2006; Hilker et al. 2007; Gregg et al. 2009; Brodie et al. 2011, B11; Mieske et al. 2013). The typical half-light radii 
of $r_{\mathrm{h}} \sim 20 \mathrm{pc}$ and luminosities of $L \sim 10^{7} L_{\odot}$ are much larger than those of globular clusters (GCs; B11), which implies that the formation process of UCDs is quite different from that of GCs. Côte et al. (2006, C06) found that there is a similarity in color, luminosity and size between UCDs and compact nuclei in a number of nucleated galaxies in the Virgo. Recent observational studies of UCDs have revealed a number of intriguing physical properties of UCDs, such as a tight color-magnitude relation for UCDs around M87 in the Virgo cluster (B11), the presence of a supermassive black hole in an UCD around M60 (Seth et al. 2014), and a young (1-2 Gyr old) stellar population in an UCD around NGC 4546 (Norris et al. 2015).

Although the formation of UCDs has been extensively discussed in the context of transformation from nucleated dwarf and spiral galaxies into naked stellar nuclei that can be identified as UCDs (e.g., Bekki et al. 2001; Pfeffer \& Baumgardt 2013), the formation of nucleated galaxies itself has not been fully understood yet. Both dissipationless merging of GCs (Fellhauer \& Kroupa 2002) and dissipative gas dynamics in the central regions of galaxies (e.g., Bekki 2007; Cole et al. 2014) have been suggested to be important for the formation and growth of stellar galactic nuclei (SGNs). Some UCDs and SGNs are observed to have high metallicities $\left(Z \sim Z_{\odot}\right)$ and young ages of less than 3 Gyr (e.g., Paudel et al. 2010; B11; Norris et al. 2015), which appears to be very hard to be explained simply by merging of metal-poor GCs and by dissipative gas dynamics in metal-poor dwarfs.

Recent observational studies of blue galaxies selected from Sloan Digital Sky Survey (SDSS) have discovered nine ultracompact blue dwarf galaxies ("UCBDs") with physical diameters less than $1 \mathrm{kpc}$ (Corbin et al. 2006). Such very compact galaxies were also reported in previous observational studies of blue compact dwarf galaxies (BCDs) by Kunth et al. (1988) and Doublier et al. (2000). The central $B$-band surface brightness of POX 186, which is the prototype UCBD, is as high as those of SGNs (Doublier et al. 2000). Furthermore, the central structures of young stellar population in some UCBDs appear to suggest that recent collision/merging events has triggered the current active star formation of these (Corbin et al. 2006). However, it is unclear whether and how these UCBDs can possibly evolve into nucleated dwarfs owing to the lack of theoretical work on this issue.

The purpose of this Letter is to propose that UCBDs can be formed from merging of low-mass, gas-rich dwarfs based on the results of numerical simulations on the physical properties of nuclear regions of dwarf-dwarf merger remnants. Bekki (2008) has suggested that galaxy merging can transform more massive gas-rich dwarf irregular galaxies into blue compact dwarfs (BCDs) and further into nucleated dwarfs. However, it did not investigate the dynamical and chemical properties of their nuclear regions. Stierwalt et al. (2015) have recently investigated physical properties of interacting dwarfs whereas Bekki 
(2015) has shown that low-mass gas-rich dwarf mergers $\left(10^{8}-10^{9} M_{\odot}\right)$ can form extremely metal-poor galaxies. However, these authors did not discuss the central regions of merging dwarfs in detail. Therefore, the present simulations are distinct from these recent ones on dwarf galaxy evolution.

\section{The model}

We perform numerical simulations of gas-rich dwarf-dwarf merging and thereby investigate the dynamical and chemical properties of the central regions of the merger remnants. We particularly investigate merging between low-mass dwarf irregular (disk) galaxies with $10^{9} \mathrm{M}_{\odot} \leq M_{\mathrm{h}} \leq 10^{10} M_{\odot}$, where $M_{\mathrm{h}}$ is the total halo mass of a dwarf, because we discuss the origins of UCBDs, UCDs, and SGNs in dwarfs. In order to simulate the time evolution of chemical abundances, star formation rates (SFRs), and gas contents, we use our original chemodynamical simulation code with dust physics that can be run on GPU machines (Bekki 2013). A dwarf galaxy consists of dark matter halo, stellar disk, and gaseous disk, and the total masses of dark matter halo, stellar disk, and gas disk are denoted as $M_{\mathrm{h}}, M_{\mathrm{s}}$, and $M_{\mathrm{g}}$, respectively. The gas mass ratio $\left(M_{\mathrm{g}} / M_{\mathrm{s}}\right)$ and baryonic mass fraction $\left(\left(M_{\mathrm{s}}+M_{\mathrm{g}}\right) / M_{\mathrm{h}}\right)$ are denoted as $f_{\mathrm{g}}$, and $f_{\mathrm{b}}$ respectively, for convenience.

The density distribution of the NFW halo (Navarro, Frenk \& White 1996) suggested from CDM simulations is adopted and the "c-parameter" $\left(c=r_{\text {vir }} / r_{\mathrm{s}}\right.$, where $r_{\text {vir }}$ and $r_{\mathrm{s}}$ are the virial radius of a dark matter halo and the scale length of the halo) and $r_{\text {vir }}$ are chosen appropriately for a given dark halo mass $\left(M_{\mathrm{h}}\right)$ by using the $c-M_{\mathrm{h}}$ relation predicted by recent cosmological simulations (Neto et al. 2007). The radial $(R)$ and vertical $(Z)$ density profiles of the stellar and gaseous disk are assumed to be proportional to $\exp \left(-R / R_{0}\right)$ with scale length $R_{0}=0.4 R_{\mathrm{s}}$ and to $\operatorname{sech}^{2}\left(Z / Z_{0}\right)$ with scale length $Z_{0}=0.02 R_{\mathrm{s}}$, respectively. Guided by observational results on $f_{\mathrm{g}}$ dependent on $M_{\mathrm{s}}$ (Papastergis et al. 2012), we investigate very gas-rich initial dwarf disks with $f_{\mathrm{g}}=1,3$, and 10 for $f_{\mathrm{b}}=0.006,0.018$, and 0.06 . The initial gaseous metallicity is set to be $\log \left(Z / Z_{\odot}\right)=-1.6$. The Kennicutt-Schmidt law $\left(\mathrm{SFR} \propto \rho_{\mathrm{g}}^{\alpha_{\mathrm{sf}}}\right.$; Kennicutt 1998) with $\alpha_{\text {sf }}=1.5$ and the threshold gas density of $\rho_{\text {th }}=100$ atoms $\mathrm{cm}^{-3}$ for star formation are adopted for modeling star formation in the present study.

We mainly investigate major merging in which two equal-mass dwarfs merge with each other to form a new dwarf. The initial distance and the pericenter distance $\left(R_{\mathrm{p}}\right)$ of two interacting/merging dwarfs are set to be $10 R_{\mathrm{s}}$ and $0.5 R_{\mathrm{s}}$, respectively. The orbital eccentricity $\left(e_{\mathrm{p}}\right)$ is set to be 1 (i.e., parabolic encounter) for all models. The spin of each galaxy in an merging pair is specified by two angles $\theta_{i}$ (in units of degrees), where suffix $i$ is used to identify each galaxy and $\theta_{i}$ is the angle between the $\mathrm{z}$ axis and the vector of the angular 
momentum of a disk. The orbital plane of a merging pair is set to be the same as the $x-y$ plane. We show the results of the models with $\theta_{1}=30$ and $\theta_{2}=45$ in the present study.

Although we have investigated the time evolution of dwarf-dwarf mergers for 1.1 Gyr in numerous models with different $M_{\mathrm{h}}, R_{\mathrm{s}}, f_{\mathrm{b}}$, and $f_{\mathrm{g}}$, we show the results of four representative models (M1-M4) with $M_{\mathrm{h}}=3 \times 10^{9} M_{\odot}$ that show typical and important behaviors of SGN and UCBD formation in the present study. We will discuss the results of other models extensively in our forthcoming papers. The fiducial model M1 has $M_{\mathrm{h}}=3 \times 10^{9} M_{\odot}, R_{\mathrm{s}}=$ $298 \mathrm{pc}, f_{\mathrm{b}}=0.018$, and $f_{\mathrm{g}}=3$, and the parameter values for other models are listed in Table 1. The total numbers of particles used for a merger model is $1.1 \times 10^{6}$, and the mass resolution for gaseous components is $4.4 \times 10^{3} \mathrm{M}_{\odot}$ in the fiducial model. The gravitational softening length for stellar and gaseous components is set to be 6 pc for the fiducial model. The softening length is different in models with different $M_{\mathrm{h}}$ and $R_{\mathrm{s}}$.

In order to estimate the $B$-band surface brightness $\left(\mu_{\mathrm{B}}\right)$ profiles of merger remnants, we use the stellar population synthesis (SSP) code, "MILES" (Vazdekis et al. 2010). Since the initial gaseous metallicity is low $\left(Z \sim 0.025 Z_{\odot}\right)$, we adopt the SSP for $Z=0.05 Z_{\odot}$ for a given age. New stars formed from gas are assumed to have the $B$-band mass-to-light-ratios $(M / L)$ of 0.09 that corresponds to a SSP with an age of 0.1 Gyr. This is reasonable and realistic, because we focus on young merger remnants dominated by young stars in the present study. Old stars are assumed to have $M / L=1.7$ that corresponds to a SSP with an age of 5 Gyr. Based on the simulated mass profiles of all stars and these $M / L$, we can estimate the $\mu_{\mathrm{B}}$ profiles of merger remnants. In the following, $T$ in a simulation represents the time that has elapsed since the simulation started.

\section{Results}

Figure 1 shows the spatial distribution of old and new stars in the merger remnant at $T=1.1$ Gyr for the fiducial model (M1). The remnant has a compact nucleus (SGN) composed almost exclusively of new stars so that it can be morphologically classified as a nucleated dwarf elliptical or spheroidal with a very blue and bright nucleus just after the merging. This formation of the blue nucleus is closely associated with the efficient inward transfer of a large amount of gas caused by energy dissipation of shocked gas during merging. As shown in Figure 2, the central surface mass density $(\Sigma)$ of new stars at $R=10$ pc can be as high as $10^{4} M_{\odot}$, which is more than an oder of magnitude higher than $\Sigma$ of old stars. Feedback effects of SNe can not completely remove the remaining gas from the nuclear region in this merger remnant so that the gas density can be also high at $T=1.1$ Gyr. 
As a result of dissipative formation of the very young SGN, the central $B$-band surface brightness $\mu_{\mathrm{B}}$ can be as high as $15 \mathrm{mag} \operatorname{arcsec}^{-2}$, which is more than five magnitudes higher than the initial $\mu_{\mathrm{B}}$ of old stars of merger progenitor dwarfs. The contribution of new stars to $\mu_{\mathrm{B}}$ becomes dominant at $R<200 \mathrm{pc}$, which is reflected on the abrupt change in the slope of the $\mu_{\mathrm{B}}$ profile around $R=200 \mathrm{pc}$. The half-light radius of the remnant is $44 \mathrm{pc}$, and $R_{25}\left(R_{27}\right)$ where $\mu_{\mathrm{B}}$ becomes $25(27)$ mag $\operatorname{arcsec}^{-2}$, is 426 (863) pc for this merger remnant. Thus, this young merger remnant can be identified as an UCBD owing to the very small $r_{\mathrm{h}}$ and the low surface brightness of the outer stellar envelope $\left(\mu_{\mathrm{B}}>25 \mathrm{mag}_{\operatorname{arcsec}}{ }^{-2}\right)$.

As shown in Figure 2, the radial $\Sigma$ and $\mu_{\mathrm{B}}$ profiles of merge remnants depend on the initial mass densities, $f_{\mathrm{g}}$, and $f_{\mathrm{b}}$. The less compact disk models, M2-M4, show lower $\Sigma$ of new stars thus systematically lower $\mu_{\mathrm{B}}$ in comparison with the fiducial model (M1). The radial $\mu_{\mathrm{B}}$ profiles for M2 and M3 are determined almost exclusively by the spatial distribution of new stars for most parts of the merger remnants. Therefore, the $\mu_{\mathrm{B}}$ profiles are relatively smooth and do not look like the observed surface brightness profiles of nucleated dwarfs (e.g., C06). On the other hand, the $\mu_{\mathrm{B}}$ profile in M4 with smaller $f_{\mathrm{g}}$ is quite similar to the observed ones for nucleated dwarfs (e.g., C06). If star formation in M4 is truncated by an external process (e.g., tidal or ram pressure stripping of gas), then this remnant can evolve into a nucleated dwarf with lower central $\mu_{\mathrm{B}}$ within the next few Gyr after fading of the SGN.

Although the $\mu_{\mathrm{B}}$ profiles are quite different between these four models, the central $\Sigma$ of gas can be rather high in all of these models. These high gaseous $\Sigma$ are due largely to the presence of nuclear gas disks surrounding SGNs in merger remnants. As shown in Figure 3, the gas disk sizes are about two times larger than $r_{\mathrm{h}}$, and the disks have outer warp-like structures (M1 and M2) and spiral arms (M4). Gas in the nuclear disks can be gradually transferred to the central regions of SGNs so that new stars can form within SGNs slowly yet steadily after dwarf-dwarf merging. These results strongly suggest that nuclear gas disks are closely related to the origin of younger ages observationally estimated for SGNs in some dwarfs and for some UCDs (e.g., B11).

The age-metallicity relation (AMR) in Figure 4 shows that the merging gasrich dwarfs can have higher metallicities in the central regions only in the later phases of merging. The merging dwarfs have lower metallicities $\left(Z<0.2 Z_{\odot}\right)$ in the early actively star-forming phases when central mass concentration starts: dwarfs at these phases might be more similar to UCBDs in terms of metallicities. They can finally have higher metallicities in the central regions after merging, mainly because chemical enrichment can proceed rapidly owing to the ejection of a large amount of metals from SNe and AGB stars that are formed efficiently 
during later starbursts in dwarf-dwarf merging. SN feedback effects can not completely suppress the formation of new stars in the central regions of merging dwarfs in the present simulations.

As a result of secondary starbursts, SGNs of the merger remnants can show large metallicity spreads of more than 0.5 dex among their stellar populations in M1-M4. As shown in Figure 4, the simulated internal metallicity spread is more remarkable in M1 with a more compact initial gas disk, because chemical enrichment proceeds longer and more efficiently in this model. The final high metallicity $\left(Z \sim Z_{\odot}\right)$ is consistent with the observed $Z$ of some SGNs and UCDs, which implies that these high-metallicity objects could have been formed from merging of gas-rich dwarf disks with higher stellar densities at a higher redshift. The metallicities of SGNs in M2, M3, and M4 with lower initial stellar densities are not so high $\left(Z<0.5 Z_{\odot}\right)$, though they are higher than typical metallicities of dwarfs.

The four models consistently show that younger stars are likely to be more metal-rich. Although the present simulations investigate only a relatively short term (1.1 Gyr) evolution of dwarf-dwarf merging, it is highly likely that the age and metallicity spread among stars of SGNs can be even larger in simulations for long-term evolution. SGNs can grow through gas accretion onto them and star formation inside SGN, as long as the nuclear gas disks can exist. The degrees of internal age and metallicity spreads in each SGN, however, would be very difficult to be precisely estimated in current spectroscopic studies for nucleated dwarfs outside the Local Group.

\section{Discussion and conclusions}

The present results provide the following four implications on the origins of UCBDs, UCDs, and nucleated galaxies. First, luminosity-weighted mean ages of stellar populations can be young in some UCDs. If dwarf-dwarf merging occurs relatively recently, the SGNs of the remnants can contain very young starbursts populations. If the remnants subsequently lose their stellar envelopes through "galaxy threshing" (i.e., tidal stripping of outer stellar envelopes of nucleated galaxies; Bekki et al 2001), then the naked nuclei can be observed as young UCDs. For example, if POX 186, which is the prototype UCBD, is stripped its outer stellar envelope through galaxy threshing, then it can be identified as a young UCD. Indeed, recent spectroscopic studies of UCDs have already found such UCDs with young or intermediate-age stellar populations (e.g., Fig. 6 in B11). The present models also predict that these apparently young UCDs have internal metallicity spreads within their stellar populations. 
Second, the observed very old $(\sim 13 \mathrm{Gyr})$ and metal-rich $\left(Z \sim Z_{\odot}\right)$ UCDs (e.g., Fig. 6 in B11) could have been formed directly from gas-rich dwarf-dwarf merging at a high redshift: These old and metal-rich UCDs can not be explained simply by the threshing scenario (Bekki et al. 2003). As shown in the present study, the remnants of mergers with higher $f_{\mathrm{g}}$ are more likely to have higher central mass densities and more diffuse outer stellar envelopes. Therefore, if dwarf-dwarf merging with possibly very high $f_{\mathrm{g}}$ can occur at a very high redshift, then the remnants can have very compact SGNs with high metallicities due to very efficient chemical enrichment. Such merger remnants could be identified as UCDs even without efficient galaxy threshing owing to the very low surface brightness of the stellar envelopes.

Third, metal-poor UCBDs with active star formation can finally become more metalrich nucleated dwarfs or UCDs with very faint stellar envelopes. Although Corbin et al. (2006) showed that UCBDs have $12+\log (\mathrm{O} / \mathrm{H})<7.65\left(\sim 0.1 Z_{\odot}\right)$, the present study suggests that at least UCBDs formed from merging can finally evolve into more metal-rich nucleated dwarfs (or UCDs) owing to continuous chemical enrichment in the high-density gas disks. Fourth, SGNs can continue to grow by converting gas of the nuclear gas disks around SGNs into new stars until the gas disks is destroyed or stripped by some external processes (e.g., ram pressure stripping). These nuclear gas disks might be responsible for the growth of massive black holes (MBHs) that coexist with SGNs in low-mass galaxies.

It should be noted here that this merger-driven SGN formation in dwarfs is only one of possible mechanisms of SGN formation, given that other mechanisms have been already proposed (e.g., Bekki et al. 2006; Antonini et al. 2012). Late-type spiral galaxies, which should not have experienced any major merger events, are observed to have nuclear star clusters or SGNs (e.g., Böker et al. 2002). Furthermore, Lotz et al (2004) found that the SGNs of dwarf ellipticals in the Virgo and Fornax clusters have colors similar to (or slightly redder than) those of GCs, which implies that metallicities of SGNs might not be so different between SGNs and GCs. Also, only less than 10\% of BCDs can be classified as UCBDs with active star formation (Doublier et al. 2000). These observations imply that merger-driven metal-rich SGN formation might not be the major mechanism of SGN formation.

Using cosmological simulations of subhalo interaction and merging in a cluster, Knebe et al (2006) demonstrated that subhalo interaction is quite important in the mass evolution of subhalos, because such interaction can tidally strip their outer envelopes. Cloet-Osselaer et al. (2014) have recently investigated merging histories of dwarfs with $M_{\mathrm{h}} \geq 10^{9} M_{\odot}$ by combining $N$-body/SPH simulations and the Press-Schechter formalism and thereby found that dwarf-dwarf merging can trigger strong star-formation episodes. Although these simulations clearly show 
the possibility of dwarf-dwarf interaction and merging, the statistics on massratios, orbital eccentricities, and pericenter distances for dwarf mergers with $M_{\mathrm{h}}=10^{9}-10^{10} M_{\odot}$ in different environments at different redshifts are yet to be extensively investigated in a cosmological context.

If dwarf-dwarf merging is more frequent at higher redshifts $(z>1)$, then young SGNs forming from such merging might be observed as unresolved point sources with $L \sim 10^{7} L_{\odot}$ owing to the very low surface brightness of the outer stellar envelopes. The present study did not discuss some key properties of SGNs, such as the small mass-ratios of SGNs to their host galaxies (e.g., Böker et al. 2002; C06; Georgiev et al. 2014), the rotational kinematics of SGNs in some galaxies like NGC 4244 (Seth et al. 2008), coexistence of massive black holes and SGNs in galaxies (e.g., Graham \& Spitler 2009). Various dynamical and hydrodynamical processes such as dynamical interaction of MBH and SGNs (e.g., Bekki \& Graham 2010) might be important for better understanding these properties of SGNs. It is therefore our future study to investigate what physical processes can be closely associated with the origin of these properties.

I am grateful to the referee, Brad Gibson, for his constructive and useful comments.

\section{REFERENCES}

Antonini, F., Capuzzo-Dolcetta, R., Mastrobuono-Battisti, A., \& Merritt, D. 2012, ApJ, 750,111

Bekki, K. 2008, MNRAS, 388, L10

Bekki, K. 2013, MNRAS, 432, 2298

Bekki, K. 2015, MNRAS, in press

Bekki, K., Couch, W. J., \& Drinkwater, M. J. 2001, ApJ, 552, L105

Bekki, K., Couch, W. J., Drinkwater, M. J., \& Shioya, Y. 2003, MNRAS, 344, 399

Bekki, K., Couch, W. J., \& Shioya, Y. 2006, ApJ, 642, L133

Bekki, K., \& Graham, A. W. 2010, ApJ, 714, L313

Brodie, J. P., Romanowsky, A. J., Strader, J., \& Forbes, D. A. 2011, AJ, 142, 199

Böker, T., et al. 2002, AJ, 123, 1389 
Cloet-Osselaer, A., De Rijcke, S., Vandenbroucke, B., Schroyen, J., Koleva, M., \& Verbeke, R. 2014, MNRAS, 442, 2909

Cole, D. R., Debattista, V. P., Erwin, P., Earp, S. W. F., \& Roskar, R. 2014, MNRAS, 445, 3352

Corbin, M. R., Vacca, W. D., Cid Fernandes, R., Hibbard, J. E., Somerville, R. S., \& Windhorst, R. A. 2006, ApJ, 651, 861

Côte, P., et al. 2006, ApJS, 165, 57

Doublier, V., Kunth, D., Courbin, F., \& Magain, P. 2000, A\&A, 353, 887

Drinkwater, M. J., Gregg, M. D., Hilker, M., Bekki, K., Couch, W. J., Ferguson, H. C., Jones, J. B., \& Phillipps, S. 2003, Nature, 423, 519

Fellhauer, M., \& Kroupa, P. 2002, MNRAS, 330, 642

Graham, A. W., \& Spitler, L. R. 2009, MNRAS, 397, 2148

Gregg, M. D., et al. 2009, AJ, 137, 498

Georgiev, I. Y., \& Böker, T. 2014, MNRAS, 441, 3570

Hilker, M., Baumgardt, H., Infante, L., Drinkwater, M., Evstigneeva, E., \& Gregg, M. 2007, A\&A, 463, 119

Jones, J. B., et al. 2006, AJ, 131, 312

Kennicutt, R. C., Jr. 1998, ApJ, 498, 541

Knebe, A, Power, C., Gill, S. P. D., \& Gibson, B. K. 2006, MNRAS, 368, 741

Kunth, D., Maurogordato, S., \& Vigroux, L. 1988, A\&A, 204, 10

Lotz, J. M., Miller, B. W., \& Ferguson, H. C. 2004, AJ, 613, 262

Mieske, S., Frank, M. J., Baumgardt, H., Lutzgendorf, N., Neumayer, N., \& Hilker, M. 2013, A\&A, 558, 14

Navarro, J. F., Frenk, C. S., \& White, S. D. M. 1996, ApJ, 462, 563

Neto, A. F., et al. 2007, MNRAS, 381, 1450

Norris, M. A., Escudero, C. G., Faifer, F. R., Kannappan, S. J., Forte, J. C., \& van den Bosch, R. C. E. 2015, MNRAS, 451, 3615 
Papastergis, E., Cattaneo, A., Huang, S., Giovanelli, R., \& Haynes, M. P. 2012, ApJ, 759, 138

Paudel, S., Lisker, T., \& Janz, J. 2010, ApJ, 724, L64

Pfeffer, J., \& Baumgardt, H. 2013, MNRAS, 433, 1997

Seth, A. C., et al. 2014, Nature, 513, 398

Stierwalt, S., Besla, G., Patton, D., Johnson, K., Kallivayalil, N., Putman, M., Privon, G., \& Ross, G. 2015, ApJ, 805, 2

Vazdekis, A., et al. 2010, MNRAS, 404, 1639 
Table 1. Description of the parameter values for the representative four models.

\begin{tabular}{lllll}
\hline \hline Model ID $^{\mathrm{a}}$ & $f_{\mathrm{b}}{ }^{\mathrm{b}}$ & $f_{\mathrm{g}}{ }^{\mathrm{c}}$ & $R_{\mathrm{s}}(\mathrm{pc})^{\mathrm{d}}$ & $r_{\mathrm{h}}(\mathrm{pc})^{\mathrm{e}}$ \\
\hline M1 & 0.018 & 3.0 & 298 & 44 \\
M2 & 0.006 & 3.0 & 473 & 53 \\
M3 & 0.018 & 10.0 & 963 & 105 \\
M4 & 0.006 & 1.0 & 963 & 53 \\
\hline
\end{tabular}

a The initial total mass of a dark matter halo $\left(M_{\mathrm{h}}\right)$ is set to be $3 \times 10^{9} M_{\odot}$ for all models.

b The initial baryonic mass fraction of a dwarf.

c The initial gas mass ratio $\left(M_{\mathrm{g}} / M_{\mathrm{s}}\right)$ of a dwarf.

$\mathrm{d}$ The initial stellar disk size of a dwarf.

e The half-light radius (in the $B$-band) of a merger remnant at $T=1.1 \mathrm{Gyr}$. 


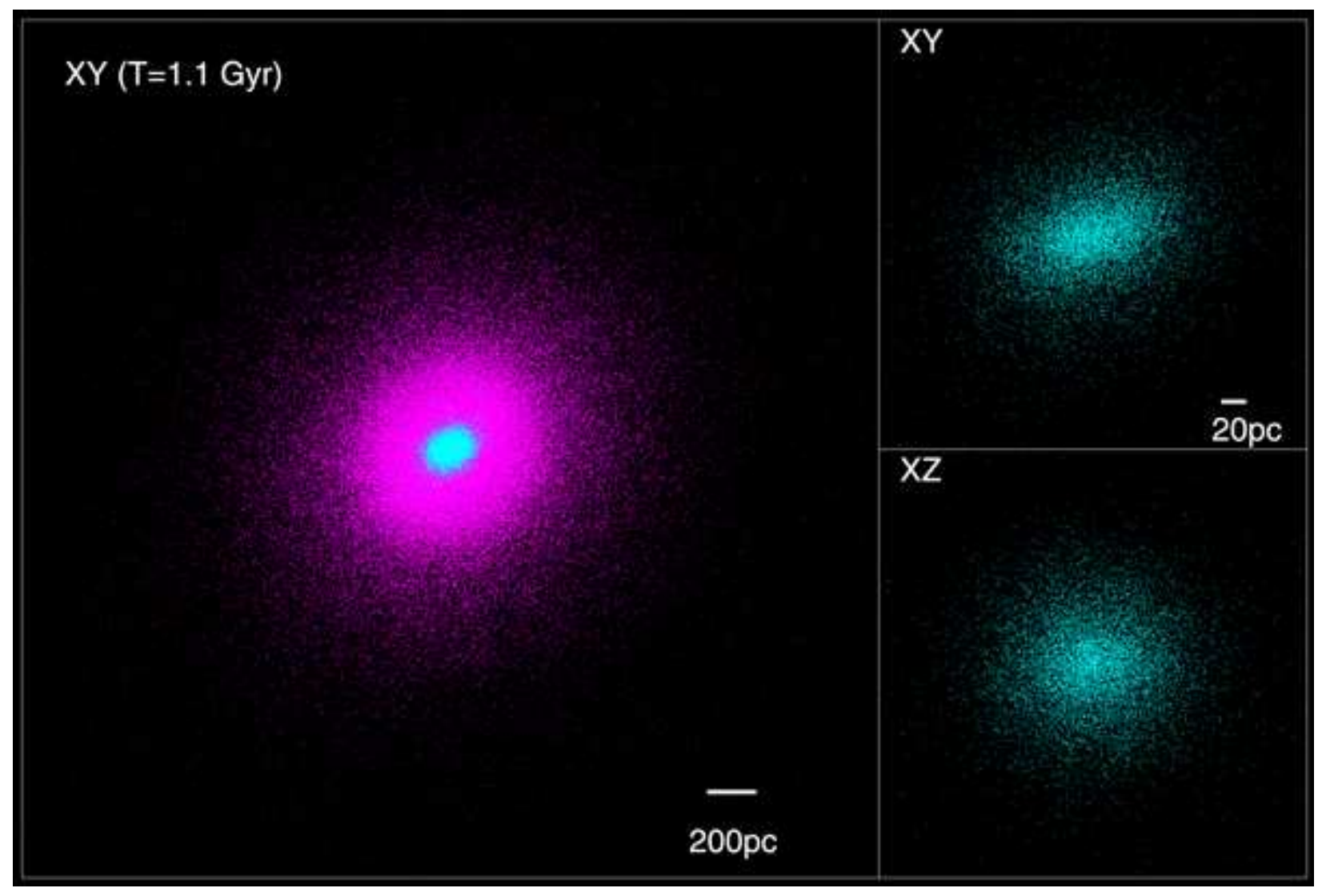

Fig. 1.- Distributions of old stars (magenta) and new ones (cyan) of the dwarf-dwarf merger remnant projected onto the $x-y$ plane on a larger scale (left) and those projected onto the $x-y$ plane (upper right) and onto the $x-z$ plane (lower right) on a smaller scale in the fiducial model (M1) at $T=1.1$ Gyr. 


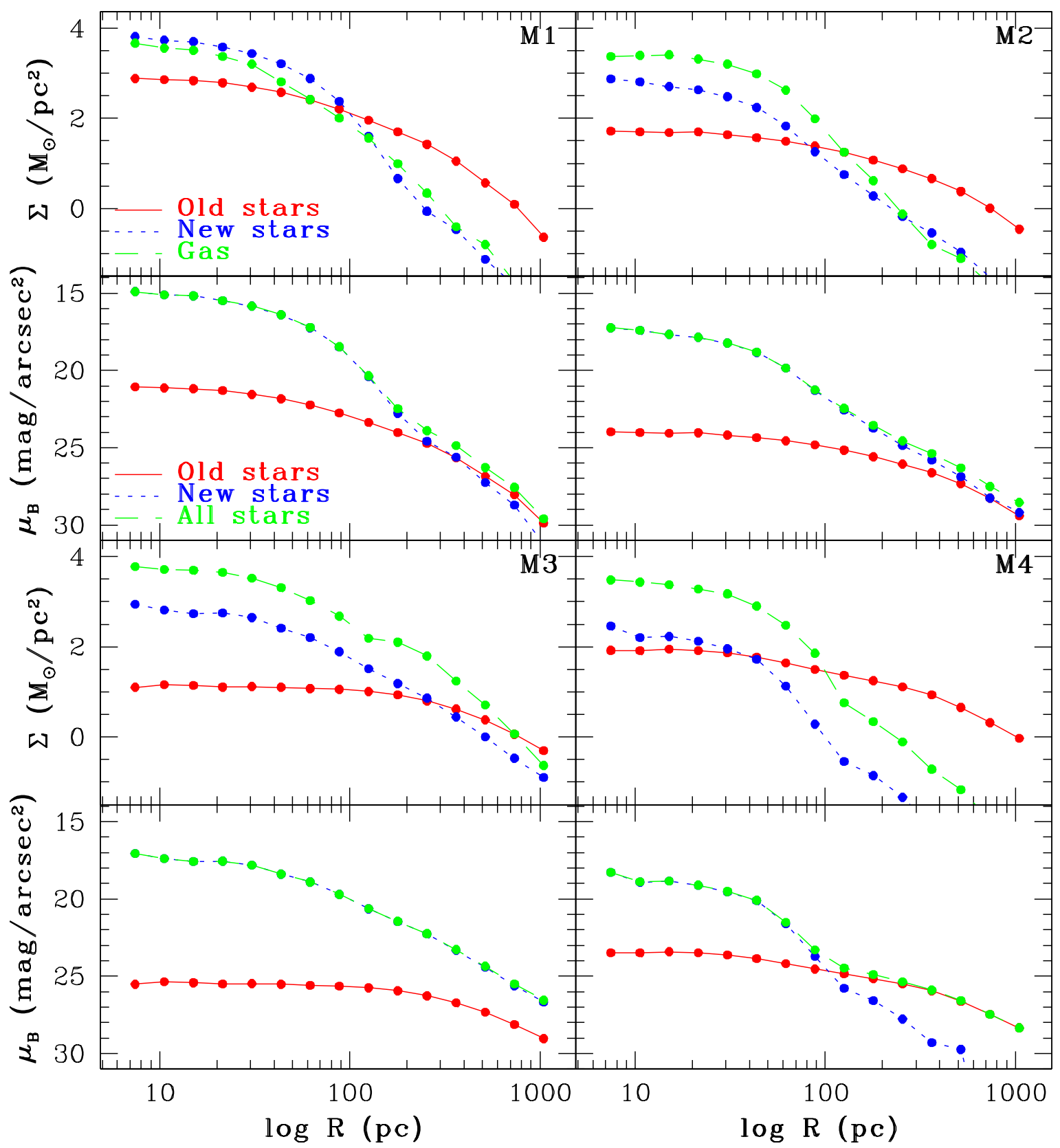

Fig. 2.- Radial surface mass density profiles $(\Sigma)$ of old stars (red solid), new stars (blue dotted), and gas (green dashed) and the radial $B$-band surface brightness profiles $\left(\mu_{\mathrm{B}}\right)$ for old stars (red solid), new stars (blue dotted), and all stars (green dashed) of the merger remnant in M1-M4 models. 


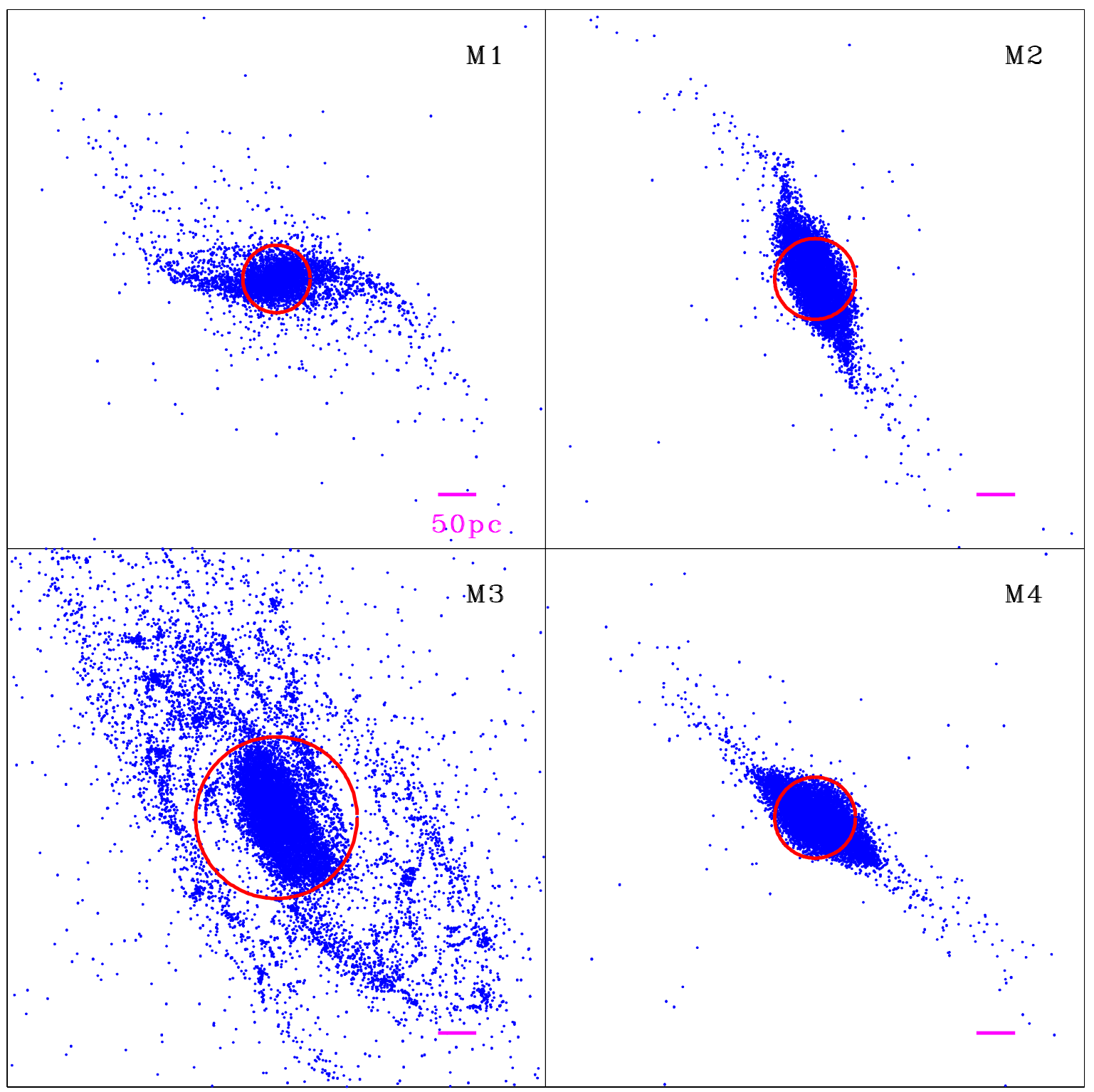

Fig. 3.- Distributions of gas around the newly formed SGNs in the remnants of dwarfdwarf mergers for the four models, M1-M4. Each red circle indicates the half-light radius of each merger remnant. 


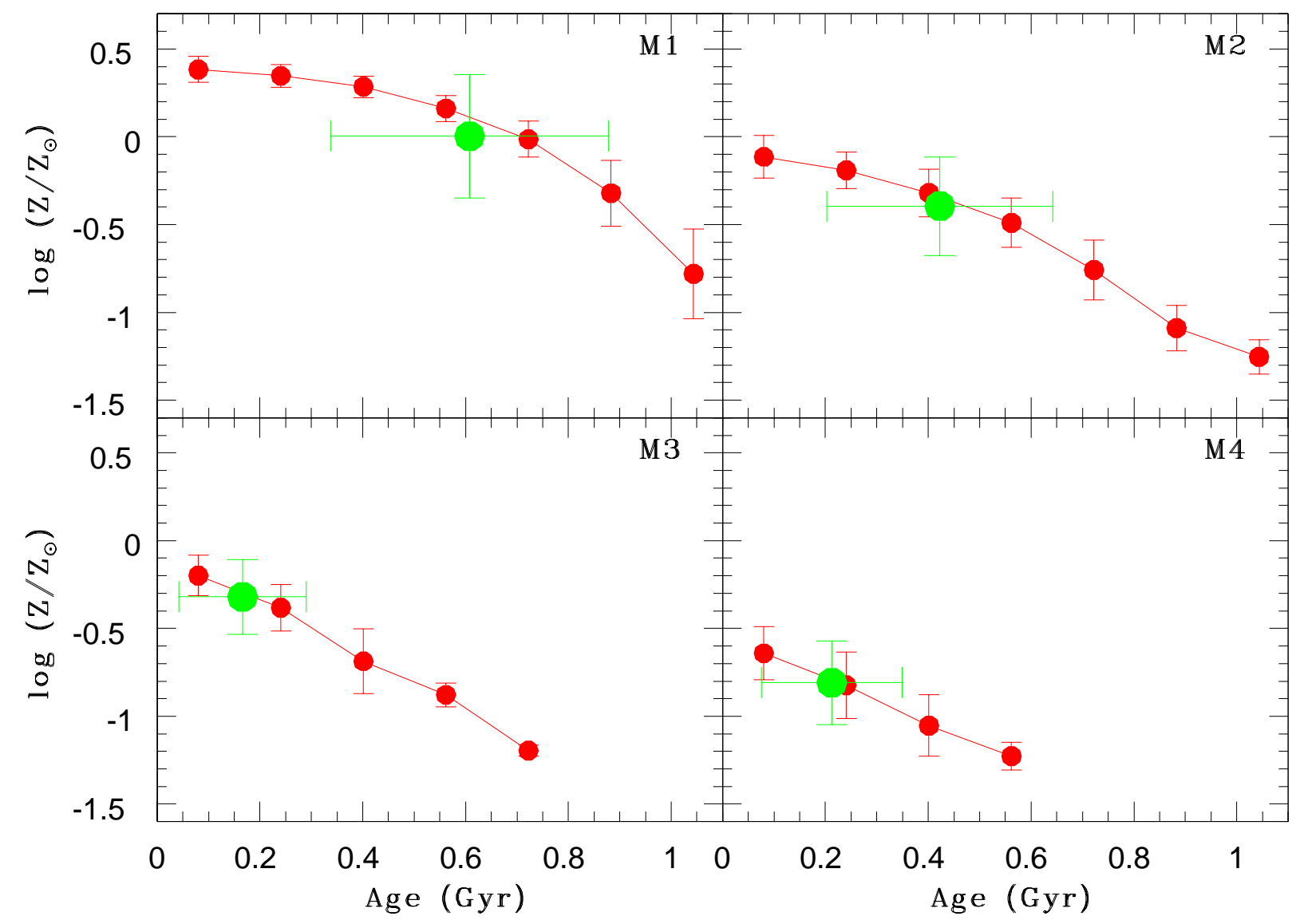

Fig. 4.- Age-metallicity relations (AMRs) of all new stars in SGNs of the merger remnants for the four models, M1-M4. The red smaller circle indicates the mean metallicity at each age bin whereas the green bigger circle indicates the mean metallicity and age of the new stars in each model. The red error bar at each age bin shows the dispersion in metallicities of new stars at each age bin. The dispersions of ages and metallicities for new stars of SGNs in each model are indicated by green error bars. The lack of data points for ages older than 0.6-0.7 Gyr in M3 and M4 is simply due to the lack of new stars formed before 0.6-0.7 Gyr ago. 